\title{
Synchronous acceleration with tapered dielectric-lined waveguides
}

\author{
F. Lemery, ${ }^{1,}$ K. Floettmann, ${ }^{1}$ P. Piot, ${ }^{2,3}$ F. X. Kärtner, ${ }^{1,4}$ and R. Aßmann ${ }^{1}$ \\ ${ }^{1}$ DESY, Notkestrasse 85, 22607 Hamburg, Germany \\ ${ }^{2}$ Department of Physics, and Northern Illinois Center for Accelerator and Detector Development, \\ Northern Illinois University DeKalb, Illinois 60115, USA \\ ${ }^{3}$ Fermi National Accelerator Laboratory, Batavia, Illinois 60510, USA \\ ${ }^{4}$ Department of Physics, University of Hamburg, Jungiusstraße 9, 20355 Hamburg, Germany
}

(Received 22 December 2017; published 25 May 2018)

\begin{abstract}
We present a general concept to accelerate nonrelativistic charged particles. Our concept employs an adiabatically-tapered dielectric-lined waveguide which supports accelerating phase velocities for synchronous acceleration. We propose an ansatz for the transient field equations, show it satisfies Maxwell's equations under an adiabatic approximation and find excellent agreement with a finite-difference timedomain computer simulation. The fields were implemented into the particle-tracking program ASTRA and we present beam dynamics results for an accelerating field with a 1-mm-wavelength and peak electric field of $100 \mathrm{MV} / \mathrm{m}$. Numerical simulations indicate that a $200-\mathrm{keV}$ electron beam can be accelerated to an energy of $\sim 10 \mathrm{MeV}$ over $\sim 10 \mathrm{~cm}$ with parameters of interest to a wide range of applications including, e.g., future advanced accelerators, and ultra-fast electron diffraction.
\end{abstract}

DOI: 10.1103/PhysRevAccelBeams.21.051302

\section{INTRODUCTION}

High-energy charged-particle accelerators have emerged as invaluable tools to conduct fundamental scientific research. Circular high energy colliders continue exploring nuclear and high-energy landscapes, searching for hints beyond the standard model. Linear accelerators capable of forming high-quality electron bunches have paved the way to bright, coherent $\mathrm{x}$-ray sources to probe ultrafast phenomena at the nanometer-scale with femtosecond resolutions in condensed matter, life science and chemistry. Accelerators have also found medical applications such as, e.g., high-resolution imaging and oncology.

Modern klystron-powered conventional accelerators incorporate radio-frequency (rf) accelerating structures optimized to provide suitable accelerating fields typically in the frequency range $f \in[0.1,10] \mathrm{GHz}$ (i.e., wavelengths respectively in the range $\lambda \in[3,0.03] \mathrm{m})$. Unfortunately, power requirements and mechanical breakdowns in accelerating cavities have limited the permissible electric fields to $E_{0} \lesssim 50 \mathrm{MV} / \mathrm{m}$, leading to $\mathrm{km}$-scale infrastructures for high-energy accelerators. These limitations have motivated the development of advanced acceleration techniques capable of supporting high accelerating fields. Accelerating structures

\footnotetext{
*francois.lemery@gmail.com
}

Published by the American Physical Society under the terms of the Creative Commons Attribution 4.0 International license. Further distribution of this work must maintain attribution to the author(s) and the published article's title, journal citation, and DOI. based on dielectric waveguides or plasmas operating in a higher-frequency regime $[\mathcal{O}(\mathrm{THz})]$ have been extensively explored in the relativistic regime.

A key challenge in accelerating low-energy nonrelativistic beams with higher frequencies stems from the difference between the beam's velocity and accelerating-mode's phase velocity. This difference leads to "phase slippage" between the beam and the accelerating field which ultimately limits the final beam energy and quality. Scaling to higher frequencies (i.e., shorter wavelengths) exacerbates the problem [1-4]. A figure of merit conventionally used to characterize the beam dynamics in the longitudinal degree of freedom during acceleration of a non-relativistic beam is the normalized vector potential $\alpha=\left(e E_{0} \lambda\right) /\left(2 \pi m c^{2}\right)$, where $e$ and $m c^{2}$ are respectively the electronic charge and rest mass, and $E_{0}$ is the time averaged accelerating field. Conventional electron photoinjectors typically operate in a relativistic regime of $\alpha \gtrsim 1$; retaining relativistic field strengths while scaling to smaller wavelengths (following $E_{0} \propto \lambda^{-1}$ ) is challenging beyond $\mathrm{rf}$ frequencies but is now routinely attained using high-power infrared lasers in plasmas operating at $f \sim 1 \mathrm{THz}$. Low- $\alpha$ acceleration with optical wavelengths, i.e., dielectric-laser acceleration (DLA) is interesting due to the foreseen compact footprints, relatively large gradients and high-repetition rates. DLA has demonstrated side-coupled grating structures $[5,6]$ which are relatively simple and allow for tapered grating periods; however, the evanescent mode inherently supports nonlinear accelerating fields, leading to defocusing forces and emittance growth. Finally, proton accelerators have established radio frequency quadrupoles (RFQs) [7] 
and drift-tube linacs with comparably long wavelengths to achieve low- $\alpha$ acceleration.

In this work, we show analytically that a longitudinallytapered dielectric-lined waveguide (DLW) can support electromagnetic fields with a longitudinally-dependent phase velocity. Therefore, by properly tailoring the spatial taper profile of the DLW, one can establish an electromagnetic field with instantaneous phase velocity $v_{p}(z)$ matching the beam velocity $\beta(z) c$ along the direction of motion $\hat{z}$ $[8,9]$. The concept is shown to be able to accelerate a nonrelativistic electron beam $(\sim 200 \mathrm{keV})$ generated out of a compact low-power rf gun to relativistic energies $\sim 10 \mathrm{MeV}$ within a few centimeters. We hypothesize an ansatz for the transient field equations supported in a tapered DLW, show they verify Maxwell's equations and validate them against a finite-difference time-domain (FDTD) electromagnetic simulation. We finally implement the transient field equations in the beam dynamics program ASTRA [10], present start-to-end simulations and validate the concept to form bright electron bunches suited for the production of attosecond x-rays via inverse Compton scattering [11]. We especially find that a single derived tapered waveguide can have a versatile range of operation, yielding electron bunches with a broad set of properties of interest for various applications.

\section{THEORY}

A cylindrical-symmetric DLW consists of a hollow-core dielectric waveguide (with relative dielectric permittivity $\left.\epsilon_{r}\right)$ with its outer surface metallized [12]. Introducing the cylindrical-coordinate system $(r, \phi, z)$, where $r$ is referenced with respect to the DLW axis along the $\hat{z}$ direction, and denoting the inner and outer radii as respectively $a$, and $b$, the electromagnetic field $(\boldsymbol{E}, \boldsymbol{H})$ associated to the accelerating $\left(\mathrm{TM}_{01}\right)$ has the following nonvanishing components:

$$
\begin{aligned}
E_{z} & =E_{0} I_{0}\left(r k_{1}\right) \sin \left(\omega t-k_{z} z+\psi\right), \\
E_{r} & =\frac{E_{0} k_{z}}{k_{1}} I_{1}\left(r k_{1}\right) \cos \left(\omega t-k_{z} z+\psi\right), \\
B_{\phi} & =\frac{\omega \epsilon_{0} \mu_{0} E_{0}}{k_{1}} I_{1}\left(r k_{1}\right) \cos \left(\omega t-k_{z} z+\psi\right),
\end{aligned}
$$

where $k_{1} \equiv \omega \sqrt{\frac{1}{v_{p}^{2}}-\frac{1}{c^{2}}}, k_{2} \equiv \omega \sqrt{\frac{\frac{e}{r}_{c^{2}}}{c^{2}} \frac{1}{v_{p}^{2}}}, k_{z}=\frac{\omega}{v_{p}}, I_{m}(\ldots)$ are the modified $m$ th order Bessel's function of the first kind, $E_{0}$ is the peak axial field amplitude, $v_{p}$ is the phase velocity, $\omega \equiv 2 \pi f$ and $\psi$ is a phase constant. In the limit $v_{p} \rightarrow c$, i.e., $\lim _{k_{1} \rightarrow 0} I_{1}\left(k_{1} r\right) / k_{1}=r / 2$ and $\lim _{k_{1} \rightarrow 0} I_{0}\left(k_{1} r\right)=1$, thus the transverse fields become completely linear and the longitudinal field becomes independent of the transverse coordinate. Conversely smaller values of $v_{p}$ result in increasingly nonlinear transverse fields and a strong dependence of $E_{z}$ on the transverse coordinate. This is a general feature of phase velocity matched modes and not restricted to the specific case discussed here. For high frequency structures the effect of nonlinearities is however exacerbated because the ratio of typical transverse beam dimensions to the wavelength is larger than in conventional rf structures.

Solutions of the characteristic equation [12] yield the allowed $\left(\omega, k_{z}\right)$ for propagating modes and depend on the DLW structure parameters $\left(a, b, \epsilon_{r}\right)$. A propagating mode must have a real-valued longitudinal component for the wave vector $k_{z}$. Correspondingly, $k_{2}$ sets a limit on the phase velocity of a propagating mode via $k_{2}<\frac{\omega n}{c}$, or $v_{p}>\frac{c}{n}$ (where $n \equiv \sqrt{\epsilon_{r}}$ is the dielectric's index of refraction). Finally, we note that the field amplitudes reduce with decreasing phase velocities $\left(v_{p} \rightarrow c / n\right)$.

We now turn to modify Eq. (1) to describe the fields associated to a tapered DLW. Specifically, we hypothesize that the transverse dimensions $(a, b)$ at a longitudinal coordinate $z$ locally determine $v_{p}$ and $E_{0}(z)$. In addition, the phase at a position $z$ should depend on the integrated phase velocity upstream of the structure. Given these conjectures, we make the following ansatz for the nonvanishing $(\boldsymbol{E}, \boldsymbol{B})$ fields

$$
\begin{aligned}
E_{z} & =E_{0}(z) I_{0}\left(r k_{1}(z)\right) \sin \left(\omega t-\int_{0}^{z} d z k_{z}(z)+\psi\right) \\
E_{r} & =\frac{E_{0}(z) k_{z}(z)}{k_{1}(z)} I_{1}\left[r k_{1}(z)\right] \cos \left(\omega t-\int_{0}^{z} d z k_{z}(z)+\psi\right) \\
B_{\phi} & =\frac{\omega \epsilon_{0} \mu_{0} E_{0}(z)}{k_{1}(z)} I_{1}\left[r k_{1}(z)\right] \cos \left(\omega t-\int_{0}^{z} d z k_{z}(z)+\psi\right),
\end{aligned}
$$

where now $k_{z}(z)$ is integrated from the structure entrance $(z=0)$ to the longitudinal coordinate $z$. The latter set of equations also introduce an explicit $z$ dependence for $E_{0}(z)$, $k_{1}(z), k_{z}(z)$. For convenience we define $\Psi(t, z) \equiv \omega t-$ $\int_{0}^{z} d z k_{z}(z)+\psi$.

In order to validate our ansatz, we check that it satisfies Maxwell's equations, starting with the Ampère-Maxwell law $\frac{\partial \boldsymbol{E}}{\partial t}=-\boldsymbol{\nabla} \times \boldsymbol{B}$ which yields

$$
\frac{1}{c^{2}} \frac{\partial E_{z}}{\partial t}=-\frac{1}{r} \frac{\partial}{\partial r}\left(r B_{\phi}\right) .
$$

Computing the right-hand side (rhs) and left-hand side (lhs) of the equation given the field components listed in Eq. (2) and making use of the identity $\frac{\partial}{\partial r} r I_{1}\left(k_{1} r\right)=k_{1} r I_{0}\left(k_{1} r\right)$ confirms that Eq. (3) is fulfilled as both sides equal $\frac{\omega}{c^{2}} E_{0} I_{0}\left(k_{1} r\right) \cos (\Psi(t, z))$.

Next, we consider Gauss' law $\boldsymbol{\nabla} \cdot \boldsymbol{E}=0$ which yields, for the fields proposed in Eq. (2),

$$
\frac{\partial}{\partial z} E_{z}=-\frac{1}{r} \frac{\partial}{\partial r}\left(r E_{r}\right)
$$


The rhs of the latter equation gives

$$
\begin{aligned}
\frac{\partial}{\partial z} E_{z}= & E_{0}\left\{-k_{z} I_{0} \cos [\Psi(t, z)]+r k_{1}^{\prime} I_{1} \sin [\Psi(t, z)]\right\} \\
& +E_{0}^{\prime} I_{0} \sin [\Psi(t, z)]
\end{aligned}
$$

while its lhs results in

$$
\begin{aligned}
-\frac{1}{r} \frac{\partial}{\partial r}\left(r E_{r}\right) & =-\frac{E_{0} k_{z} \cos [\Psi(t, z)]}{k_{1} r} \frac{\partial}{\partial r}\left[r I_{1}\left(k_{1} r\right)\right] \\
& =-k_{z} E_{0} I_{0}\left(k_{1} r\right) \cos [\Psi(t, z)] .
\end{aligned}
$$

Equations (5) and (6) are generally not equal unless the tapering of the DLW is sufficiently slow or adiabatic,

$$
\left|\frac{r k_{1}^{\prime} I_{1}\left(k_{1} r\right)}{k_{z} I_{0}\left(k_{1} r\right)}\right| \ll 1, \quad \text { and } \quad\left|\frac{E_{0}^{\prime}}{E_{0} k_{z}}\right| \ll 1 .
$$

Equations (7) are independent of $\epsilon_{r}$ and present general conditions to the evolution of a traveling mode in a tapered waveguide which may also be of interest to plasma acceleration with, e.g., smaller field gradients and potentially higher repetition rates.

\section{BEAM DYNAMICS}

Insofar, our discussion has solely concentrated on the electromagnetic aspect of the problem. Let us now consider the beam dynamics of a charged particle accelerating in a tapered DLW. The requirement for continuous synchronous acceleration imposes $v_{p}(z)=\beta(z) c$. The longitudinal phase space dynamics is described by the coupled ordinary differential equations,

$$
\begin{aligned}
& \frac{\partial z}{\partial t}=\beta c, \\
& \frac{\partial \beta}{\partial t}=\frac{e E_{0}(z)}{\gamma^{3} m c} I_{0}\left(k_{1} r\right) \sin (\Psi) .
\end{aligned}
$$

In addition to synchronous acceleration, the transversedynamics plays a crucial role in the formation of bright electron beams. The transverse force can be calculated from the Lorentz force,

$$
\begin{aligned}
F_{r} & =e\left(E_{r}-\beta c B_{\phi}\right) \\
& =e E_{0}\left(\frac{1}{\beta_{p}}-\beta\right) k_{0} \frac{I_{1}\left(k_{1} r\right)}{k_{1}} \cos (\Psi),
\end{aligned}
$$

where $\beta_{p} \equiv v_{p} / c$ is the normalized phase velocity. For synchronous acceleration $\left(\beta=\beta_{p}\right)$, the latter equation simplifies to

$$
F_{r}=e E_{0} \frac{k_{0}}{\gamma^{2} \beta} \frac{I_{1}\left(k_{1} r\right)}{k_{1}} \cos (\Psi),
$$

implying transverse defocusing forces for $\Psi \in[-90,0]$ deg (i.e., the compression phase where the bunch tail experiences a stronger longitudinal field than the head) and transverse focusing forces for $\Psi \in[-180,-90]$ deg and no transverse force on-crest at $\Psi=-90 \mathrm{deg}$. We note that the amplitude of the force is increased by a combination of the particle and the phase velocity [Eq. (9)], while the nonlinearity of the field is a result of the matching to the phase velocity alone. For $v_{p} \sim c$, as, e.g., in conventional rf guns, [Eq. (9)] reduces to $F_{r}=e E_{0} k_{0} /\left(\gamma^{2}(1+\beta)\right) r / 2$; the transverse fields are linear in $r$ and the longitudinal field is independent of the radial coordinate. In the matched case however, strong and nonlinear fields appear at low energies, which in combination with the r-dependence of $E_{z}$, strongly affect the beam dynamics. The transverse emittance is an important figure of merit which characterizes the phase-space density of the beam defined as $\varepsilon_{r} \simeq \varepsilon_{u}=\frac{1}{m c}\left[\left\langle u^{2}\right\rangle\left\langle p_{u}^{2}\right\rangle-\left\langle u p_{u}\right\rangle\right]^{1 / 2}$ where $\langle\ldots\rangle$ is the statistical averaging over the beam distribution. Therefore at injection, the transverse beam size $\sigma_{r}$ should be minimized to mitigate emittance and energy spread dilutions.

We now describe a start-to-end simulation considering a driving field with $\lambda=1 \mathrm{~mm}(f=300 \mathrm{GHz})$ and $E_{0}=$ $100 \mathrm{MV} / \mathrm{m}$ corresponding to $\alpha \simeq 0.03$. A $\mathrm{C}++$ program was developed to integrate the equations of motion (Eqs. (8) for one electron given the set of initial conditions: electron injection energy, wavelength, peak accelerating field, and DLW geometry. In parallel, the characteristic equation is solved to derive the appropriate taper. Consequently, an electron injected on crest will not experience any phaseslippage through the structure. Additionally, scaling the accelerating field $E_{0} \rightarrow \eta E_{0}$ offsets the point of zero phase-slippage by an amount $\delta \Psi=\arcsin (1 / \eta)$. We specialize our study to the case where the DLW has a constant inner radius $a$ and devise the outer radius $b(z)$ to ensure

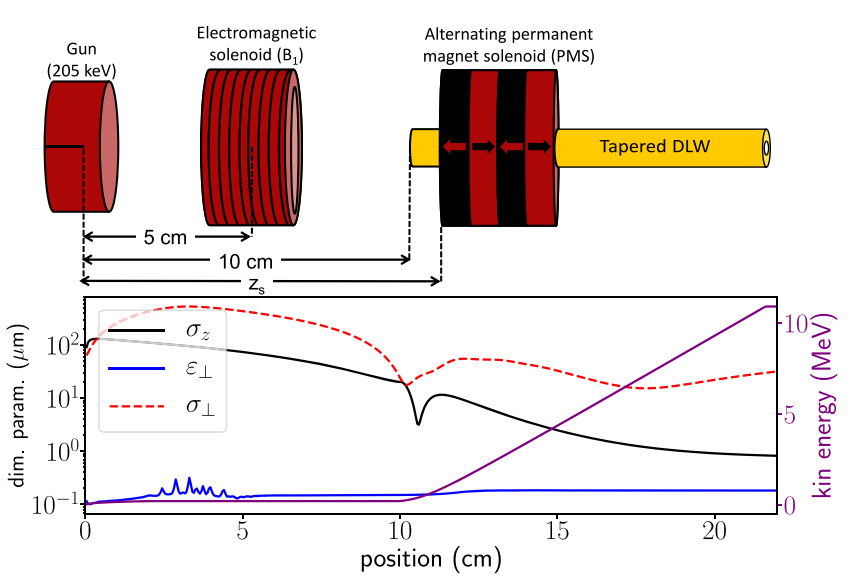

FIG. 1. Diagram of the accelerator concept (top) and corresponding evolution of the bunch's transverse emittance $\left(\varepsilon_{r}\right)$, rms transverse beam size $\left(\sigma_{r}\right)$, longitudinal bunch length $\left(\sigma_{z}\right)$ (all left axis) and the kinetic energy (right axis) along the accelerator beamline (bottom). The example corresponds to an operating point $\left(\phi, E_{0}\right)=(79.3 \mathrm{deg}, 106.875 \mathrm{MV} / \mathrm{m})$; see text for details. 

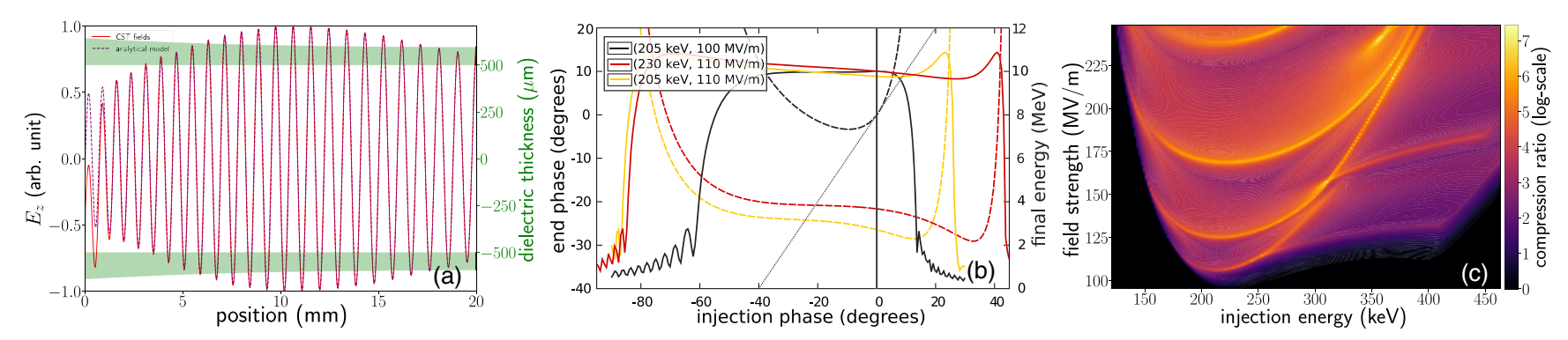

FIG. 2. (a) Geometry of the dielectric-layer tapering (green shaded area, right axis) over the entrance of the structure; the initial dielectric thickness is $143 \mu \mathrm{m}\left(v_{p}=0.7 c\right)$ and asymptotically approaches $91 \mu \mathrm{m}\left(v_{p}=c\right)$. In addition we show the comparison between our analytic field ansatz with FDTD code CST-Mws over the first $20 \mathrm{~mm}$. (b) Final energy (solid traces, left column) and end phase (dashed lines, right column) as a function of injection phase for various accelerating gradients and initial kinetic energies. The black diagonal dashed line shows $\phi_{e}=\phi_{i}$, intersections with the phase portraits indicate zero phase-slippage. (c) The compression ratio between the injection phase and end phase, $\Delta \phi_{i} / \Delta \phi_{e}$ in log-scale as a function of injection energy and accelerating gradient for an input bunch length spanning $60 \mathrm{deg}\left(\Delta \phi_{i}=60 \mathrm{deg}\right.$.)

synchronous acceleration throughout the DLW. The DLW is taken to be made of quartz $\left(\epsilon_{r}=4.41\right)$ with length $L=11.5 \mathrm{~cm}$, and $a=0.5 \mathrm{~mm}$. This choice of material limits the phase velocity to values $v_{p}>c / n \sim .48 c$ thereby requiring an injection energy $>70 \mathrm{keV}$. The group velocity of $\sim 0.5 \mathrm{c}$ and length of the structure dictate a required pulse length of $\sim 383 \mathrm{ps}$ corresponding to a pulse energy of $7.2 \mathrm{~mJ}$ for $E_{0}=100 \mathrm{MV} / \mathrm{m}$.

We consider a compact, low-power, field-enhanced S-band $(f=3 \mathrm{GHz})$ gun [13] with a photocathode as our electron source. The gun is operated off-crest to generate short $\sigma_{z} \sim 20 \mu \mathrm{m}, 205 \mathrm{keV}$ electron bunches at the DLW entrance with a total charge $Q=100 \mathrm{fC}$; our simulation used 20,000 macroparticles. An electromagnetic solenoid with variable peak axial magnetic field $B_{1}$ is located $5 \mathrm{~cm}$ downstream of the cathode and focuses the bunch into the DLW structure positioned $10 \mathrm{~cm}$ from the cathode. To control the strong defocusing forces [Eq. (9)] during the early stages of acceleration, a 4-slab, $\sim 4 \mathrm{~cm}$ alternatingpermanent-magnet solenoid [14] with a maximum axial

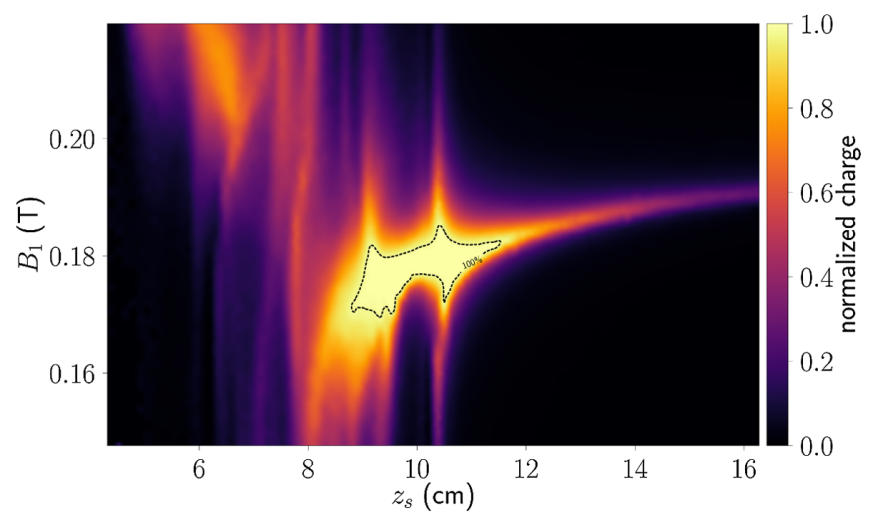

FIG. 3. Charge fractional transmission through the structure as a function $B_{1}$ and $z_{s}$ for injection parameters $\left(\mathcal{E}_{i}, E_{z}\right)=$ (205 keV, $105.8 \mathrm{MV} / \mathrm{m}$ ) corresponding to a maximum bunch compression point from Fig. 2(c). A black dashed line encompasses $100 \%$ transmission. field $B_{2}=1.5 \mathrm{~T}$ surrounds part of the DLW and is located at a distance $z_{s}$ from the cathode; see Fig. 1 (top). This setup was not globally optimized.

At the entrance of the structure the matched phase velocity is $v_{p}=0.7 c$ and the accelerating gradient is reduced to $E_{0} \sim 20 \mathrm{MV} / \mathrm{m}$; the adiabatic condition from Eq. (7) for $r=100 \mu \mathrm{m}$ at $z=0$ gives 0.0017 and approaches $10^{-7}$ toward the end of the structure. For completeness we use the FDTD program CST MWS [15] to simulate the field propagation for the first $2 \mathrm{~cm}$ of the DLW where the majority of the taper occurs; here we simulated a Gaussian THz pulse with $1 \%$ bandwidth, while our simulation in ASTRA utilizes a flat-top pulse. The simulated fields are in excellent agreement with our semianalytical field; see Fig. 2(a). Some discrepancies arise at the entrance and exit of the structure due to transient effects not included in our model.

We can gain some significant insight into the longitudinal dynamics with a single electron; we illustrate the energy gain and end phase as a function of initial phase in Fig. 2(b). Generally, larger accelerating gradients and injection energies than the matched conditions increase the longitudinal acceptance of the structure. Plateaus in the end phases suggests bunch compression across the flat injection phase width. In Fig. 2(c) we show the resulting compression ratio in $\log$-scale, $\frac{\Delta \phi_{i}}{\Delta \phi_{e}}$, as a function of initial energy $\mathcal{E}_{i}$ and field strength $E_{z}$ for an input bunch spanning $60 \mathrm{deg}$. The phase trajectory through the structure is determined by $\mathcal{E}_{i}, E_{z}$, and $\phi_{i}$; changing these parameters will alter the phase trajectory and impact the forces experienced by the bunch along the structure; this implies that a single structure has a very broad range of operational capabilities.

The transverse matching into and through the structure essentially depends on the balance between the transverse defocusing forces from the DLW and focusing optics from the solenoids. Different phase trajectories will generally have different transverse forces along the structure. We illustrate the transmission through the structure as a 

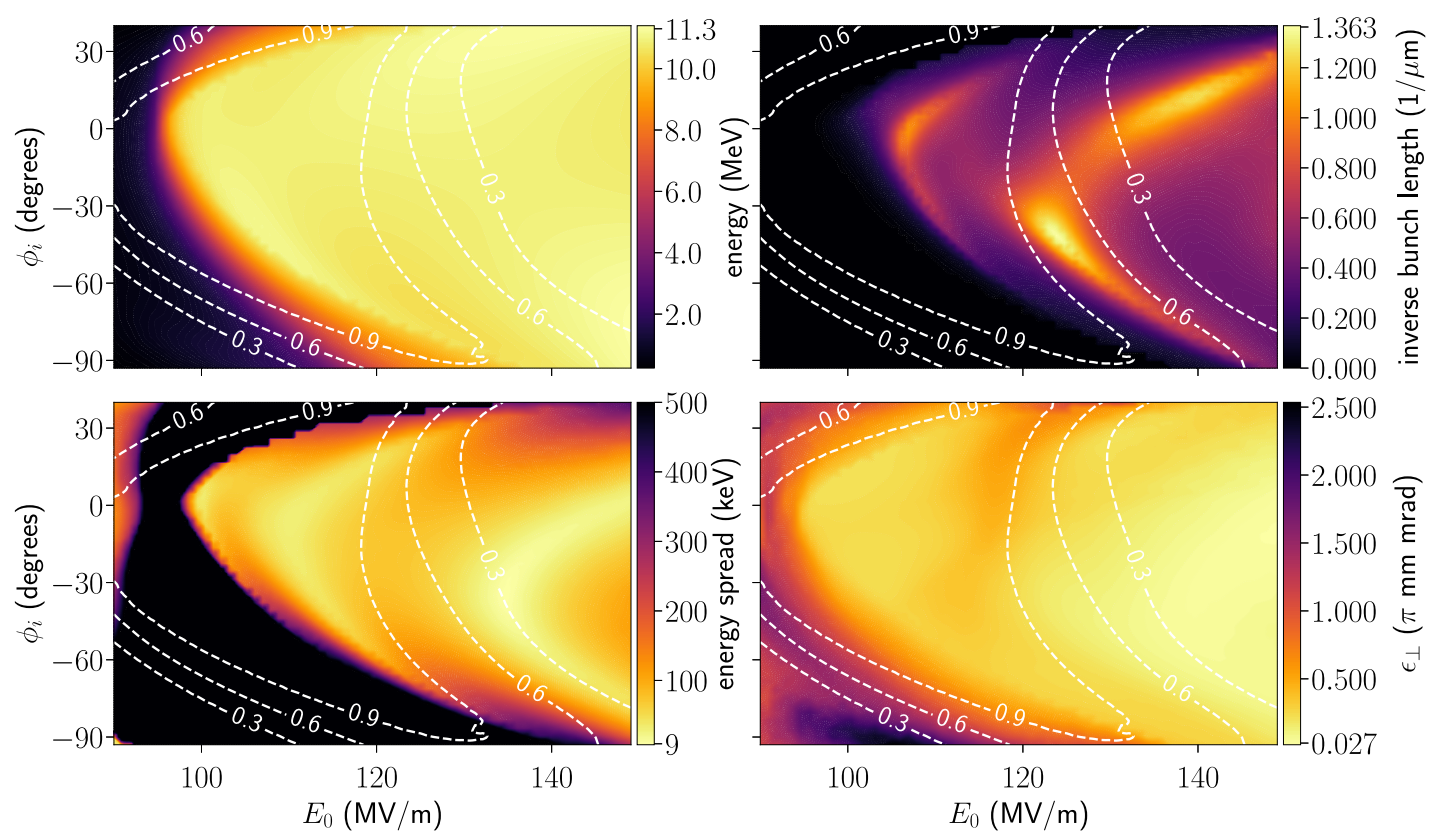

FIG. 4. Final bunch energy, inverse bunch length, energy spread, and normalized transverse emittance for the matched case, $\left(B_{1}, z_{s}\right)=(0.179 \mathrm{~T}, 10.5 \mathrm{~cm})$. In each case we overlay the final bunch charge as white contour levels for $0.3,0.6$, and 0.9 charge transmission. While all final energies are approximately equal $(\sim 11 \mathrm{MeV})$, the structure allows for the production of widely-tunable electron beams.

function of our matching optics $B_{1}$ and $z_{s}$ in Fig. 3 associated to a local compression maximum $\left(\mathcal{E}_{i}, E_{z}\right)=$ (205 keV, 105.8 MV/m). To accommodate such an injection energy, we accordingly minimize the bunch length by choosing an appropriate field strength and injection phase in the gun, $E_{\text {gun }}=113.55 \mathrm{MV} / \mathrm{m}, \phi_{\text {gun }}=217 \mathrm{deg}$. Larger acceptances allow less stringent requirements on the beam matching and allows a larger operational range for the same matching point.

Finally, we explore the beam dynamics of the structure for a matching point in Fig. $3,\left(B_{1}, z_{s}\right)=(0.179 \mathrm{~T}, 10.5 \mathrm{~cm})$ and show the resulting final energy, energy spread, inverse bunch length, and transverse emittance for scans over $\left(\phi, E_{0}\right)$ in Fig. 4. In each figure we include white contour lines representing the final bunch charge; in cases with large offsets to the originally matched conditions, e.g., large gradients, the larger defocusing forces leads to internal collimation, which in some instances leads to, e.g., reduction in emittance. The relationship between these final beam parameters can be viewed with the 6 dimensional (6D) brightness, $B_{6 D}=\frac{Q}{\epsilon_{x} \epsilon_{y} \epsilon_{z}}$, a figure of merit describing the phase space density; see Fig. 5. The largest brightness corresponds to an acclerating gradient $\sim 20 \%$ larger than the design amplitude. One should of course investigate and optimize a structure based on the desired final bunch characteristics and injection constraints; however the large operational range of a single structure implies broad and stable operation for a single matching point.

Some notable operating points include, $\left(\sigma_{z}, \epsilon_{r}, \sigma_{E}, Q\right)=$ $(1.2 \mu \mathrm{m}, 250 \mathrm{~nm}, 47.6 \mathrm{keV}, 100 \mathrm{fC}) \quad$ for $\quad\left(\phi, E_{0}\right)=$
( $-10.7 \mathrm{deg}, 106.875 \mathrm{MV} / \mathrm{m})$. The shortest bunch length achieved in our scans for the associated structure was $\left(\sigma_{z}, \epsilon_{r}, \sigma_{E}, Q\right)=(730 \mathrm{~nm}, 158 \mathrm{~nm}, 83 \mathrm{keV}, 80 \mathrm{fC})$ for the operational point $\left(\phi, E_{0}\right)=(-41.2 \mathrm{deg}, 123.75 \mathrm{MV} / \mathrm{m})$; smaller energy spreads can be reached also, at the expense of other final parameters. Finally we illustrate the longitudinal phase space (LPS) and current profile for the case of largest brightness, i.e., $\left(\phi, E_{0}\right)=(-44 \mathrm{deg}, 125.5 \mathrm{MV} / \mathrm{m})$ in Fig. 6 which experiences $87 \%$ charge transmission. We note the long tails increase the $\sigma_{z}$; the full width at half maximum (FWHM) is $\sim 0.57 \mu \mathrm{m}$ and contains $\sim 47 \mathrm{fC}$ of charge. A shorter bunch at injection or longer wavelengths would ameliorate the final bunch compression.

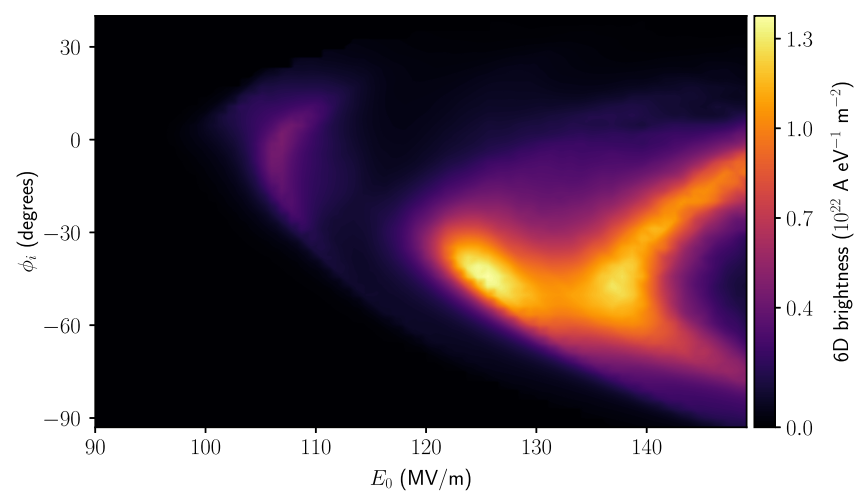

FIG. 5. The final beam brightness $B_{6 D}=\frac{Q}{\epsilon_{x} \epsilon_{y} \epsilon_{z}}$, is illustrated over $\left(\phi, E_{0}\right)$. The maxima correspond very closely with minimum bunch lengths but differentiations arise from charge losses and, e.g., beam dilution. 


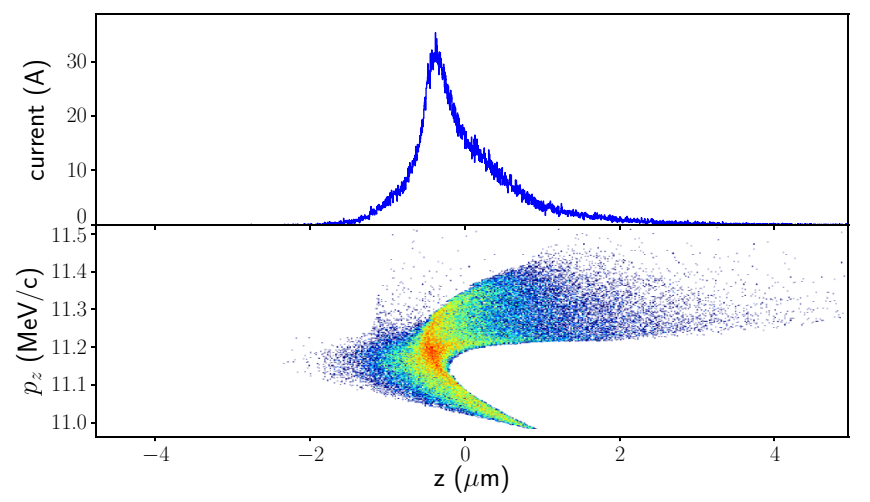

FIG. 6. The longitudinal phase space (LPS) and corresponding current profile is illustrated for the operational point yielding the largest beam brightness, $B_{6 D}=1.3 \times 10^{22}$, for $\left(\phi, E_{0}\right)=$ $(-41.2 \mathrm{deg}, 123.75 \mathrm{MV} / \mathrm{m})$. The maximum peak current of the bunch is $\sim 35 \mathrm{~A}$.

\section{DISCUSSION}

In summary, we have proposed adiabatically-tapered dielectric-lined waveguides to accelerate and manipulate low-energy charged particles with nonrelativistic field strengths, i.e., in the low- $\alpha$ regime. We hypothesized an ansatz for the transient field equations and support them with Maxwell's equations and computer simulation. We implemented the fields directly into ASTRA and performed beam dynamics simulations for a low-energy electron bunch accelerating in a $1 \mathrm{~mm}$ field with $100 \mathrm{MV} / \mathrm{m}$. The derived structure supports nonphase-slipping trajectories for various input powers; offsets in the initial parameters leads to very similar final energies but with a wide variety of other bunch properties, notably small bunch lengths and energy spreads. We presented a very simple beam matching scheme to accommodate the strong-defocusing forces in the early stages of acceleration. Our derivation of Eq. (7) is independent of $\epsilon_{r}$ and is therefore a more general relation to the evolution of a traveling mode and may appeal to, e.g., plasma acceleration with smaller field gradients and potentially higher repetition rates via tapered density profiles.

The proposed setup was not globally optimized. The beam matching could be improved via the positions and strengths between the elements to further minimize emittance growth for a given charge. Likewise, larger charges could be accelerated with higher injection energies, longer accelerating wavelengths, and larger accelerating field strengths. Acceleration with lower initial energies can be achieved with larger dielectric permittivities, and could possibly realize a stand-alone relativistic electron source. A performance analysis given fabrication imperfections should be studied.

\section{ACKNOWLEDGMENTS}

F. L. is thankful to DESY's accelerator division for support to continue this ongoing work. This project is funded by the European Union's Horizon 2020 Research and Innovation programme under Grant Agreement No. 730871 and also supported by the European Research Council (ERC) under the European Union's Seventh Framework Programme (FP/2007-2013)/ERC AXSIS Grant agreement No. 609920. P. P. is sponsored by U.S. NSF Grant No. PHY-1535401. We acknowledge the use of DESY's high-performance computing center MAXWELL and are grateful to F. Schluenzen for support. We thank M. Fakhari for providing the S-band gun design.

[1] K.-J. Kim, Rf and space-charge effects in laser-driven rf electron guns, Nucl. Instrum. Methods Phys. Res., Sect, A 275, 201 (1989).

[2] J. Rosenzweig and E. Colby, Charge and wavelength scaling of RF photoinjector designs, AIP Conf. Proc. 335, 724 (1995).

[3] P. Piot, L. Carr, W. S. Graves, and H. Loos, Subpicosecond compression by velocity bunching in a photoinjector, Phys. Rev. ST Accel. Beams 6, 033503 (2003).

[4] K. Floettmann, rf-induced beam dynamics in rf guns and accelerating cavities, Phys. Rev. ST Accel. Beams 18, 064801 (2015).

[5] T. Plettner, P. Lu, and R. L. Byer, Proposed few-optical cycle laser-driven particle accelerator structure, Phys. Rev. ST Accel. Beams 9, 111301 (2006).

[6] J. Breuer and P. Hommelhoff, Laser-Based Acceleration of Nonrelativistic Electrons at a Dielectric Structure, Phys. Rev. Lett. 111, 134803 (2013).

[7] I. M. Kapchinskii and V. A. Teplvakov, A linear ion accelerator with spatially uniform hard focusing, Prib. Tekh. Eksp. 2, 19 (1970).

[8] F. Lemery and P. Piot, in Proceedings of the 6th International Particle Accelerator Conference (IPAC'15), Richmond, VA, USA, 2015 (JACoW, Geneva, Switzerland, 2015), p. 2664.

[9] F. Lemery, K. Floettmann, P. Piot, and F. X. Kaertner, in Proc. of International Particle Accelerator Conference (IPAC'17), Copenhagen, Denmark, 2017, 8 (JACoW, Geneva, Switzerland, 2017), p. 2861.

[10] K. Floettmann, Astra - a space charge tracking algorithm (2011).

[11] F. X. Kaertner et al., AXSIS: Exploring the frontiers in attosecond X-ray science, imaging and spectroscopy, Nucl. Instrum. Methods Phys. Res., Sect. A 829, 24 (2016); 2nd European Advanced Accelerator Concepts Workshop EAAC 2015.

[12] S. Frankel, $T M_{0,1}$ Mode in Circular Wave Guides with Two Coaxial Dielectrics, J. Appl. Phys. 18, 650 (1947).

[13] H. Daoud, K. Floettmann, and D. Miller, Compression of high-density $0.16 \mathrm{pC}$ electron bunches through high field gradients for ultrafast single shot electron diffraction: The Compact RF Gun, Structural Dynamics 4, 044016 (2017).

[14] T. Gehrke, Design of permanent magnetic solenoids for REGAE, Master's thesis, University of Hamburg, 2013.

[15] Computer simulation technology (cst). 